\title{
URUGUAY 2013: UN BALANCE PREELECTORAL
}

\author{
Uruguay 2013: A pre-electoral analysis
}

\author{
SANTIAGO LÓPEZ CARIBONI \\ Departamento de Ciencias Sociales y Políticas, Universidad Católica del Uruguay
}

\section{JUAN ANDRÉS MORAES}

Departamento de Ciencia Política, Universidad de la República

\section{RESUMEN}

Este artículo analiza la coyuntura política de Uruguay durante 2013. La primera sección muestra una evolución del contexto socioeconómico en el que ha navegado Uruguay durante los últimos cinco años, haciendo hincapié en los resultados recientes en algunas áreas específicas de política pública. La segunda sección se concentra en la actividad del Poder Ejecutivo, como el principal agente en la fijación de la agenda de gobierno, mediante la actividad de los gabinetes y la gestión del liderazgo presidencial en el sistema político. La tercera sección está dedicada a la actividad del Poder Legislativo, no solo como agente colegislador junto al Poder Ejecutivo, sino como institución dedicada al contralor de las actividades del Ejecutivo. La cuarta sección se detiene en la opinión pública para analizar la evaluación del gobierno y la evolución de la intención de voto para las elecciones de 2014. La quinta sección analiza el acuerdo electoral entre Blancos y Colorados en Montevideo, de cara al ciclo electoral de 2014-2015. La última sección concluye y repasa algunos escenarios esperables de 2014.

Palabras clave: Uruguay, Mujica, coyuntura, gobierno, elecciones.

\begin{abstract}
This article analyzes Uruguayan politics in 2013. The first section shows the country's social and economic context over the last five years emphasizing some specific public policy outputs. The second section focuses on the Executive Power as the main agenda setter acting through the ministerial cabinet and the presidential leadership. The third section is dedicated to the Legislative Power both in terms of its legislative productivity along with the Executive Power and its control over the Presidency. The fourth section concentrates on public opinion analyzing the evolution of government approval and the electoral race for the next year. The fifth section pays attention to the electoral agreement between the National Party and Colorado Party in Montevideo to compete for that municipality in 2014. Last section concludes and overviews some scenarios for the upcoming year.
\end{abstract}

Key words: Uruguay, Mujica, outlook, government, elections. 


\section{INTRODUCCIÓN}

Uruguay es el único gobierno de izquierda en América Latina que combina una agenda de políticas de corte libertario con una clara orientación hacia la economía de mercado, sin descuidar uno de los estados de bienestar más desarrollados del continente. Esta configuración ha tenido como pivot a un presidente bien reputado en la escena regional e internacional, pero que a nivel doméstico ha visto erosionar su popularidad debido a la evolución de algunos resultados en áreas de política pública de gran impacto social, ciertos problemas en la gestión de la agenda del gobierno, la dinámica interna de su partido y el relacionamiento con la oposición. A cuatro años de gobierno del presidente Mujica, Uruguay enfrenta dos procesos marcados por la incertidumbre. Por un lado, el país se encuentra por primera vez en nueve años frente un marcado contexto desfavorable en el plano económico regional e internacional. Por otro, el proceso político está signado por las elecciones internas y la elección nacional de octubre de 2014. Estos dos componentes del contexto no solo han definido parte importante de 2013, sino que además contribuirán a explicar algunos eventos observables durante 2014.

\section{PANORAMA SOCIOECONÓMICO}

La economía uruguaya muestra algunos cambios en el último bienio. En primer lugar, el crecimiento económico de los últimos diez años bajó de ritmo en 2012 y 2013. En 2012 la variación anual del PIB había alcanzado el 3,9\%, mientras que en 2013 se estima que el crecimiento llegó al 3,8\%. Mientras que el contexto internacional ha dejado de contribuir sustancialmente con el crecimiento económico, la economía uruguaya depende cada vez más del consumo interno. El problema principal es que el mercado doméstico ya no es capaz de mantener el crecimiento sin generar otros desequilibrios como las presiones inflacionarias. Aun así, las expectativas de crecimiento para el 2014 son similares a las de 2013. La Figura 1 muestra la evolución del crecimiento del PIB anual entre 2005 y 2013. De acuerdo con los analistas económicos, uno de los límites del crecimiento de la economía uruguaya es que el aumento del capital humano es cada vez más costoso y el resto de los factores productivos están cerca de su límite (CINVE, 2013).

En segundo lugar, la inflación fue el principal desajuste macroeconómico que enfrentó el Uruguay en 2013. Mientras que el rango-meta de inflación definido por el gobierno para ese año fue de entre $4 \%$ y $6 \%$, el incremento de precios acumulado anual alcanzó el $8,52 \%$ (ver Figura 1). Los rubros que explican mayormente el incremento de precios son los menos volátiles y los de mayor consumo de los hogares: alimentos y alquileres. El gobierno ha intentado por distintas vías mantener el nivel de precios dentro del rango definido mediante cierta contracción monetaria y el control directo de precios. Por ejemplo, las tarifas de los servicios públicos como la electricidad y el suministro de agua aumentaron por debajo de la inflación. Aun así el índice de precios global fue muy simi- 
Figura 1. Principales indicadores macroeconómicos

Crecimiento PIB

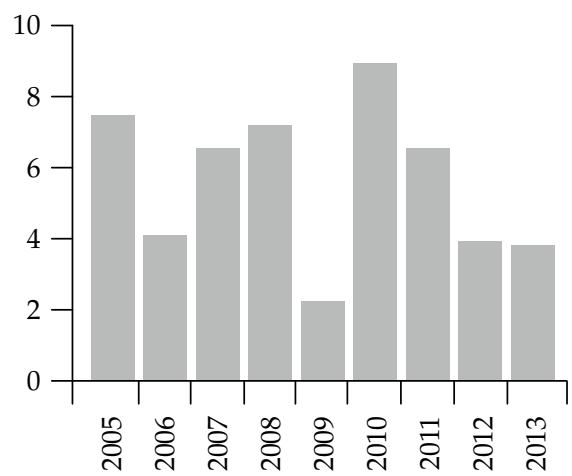

Salario real

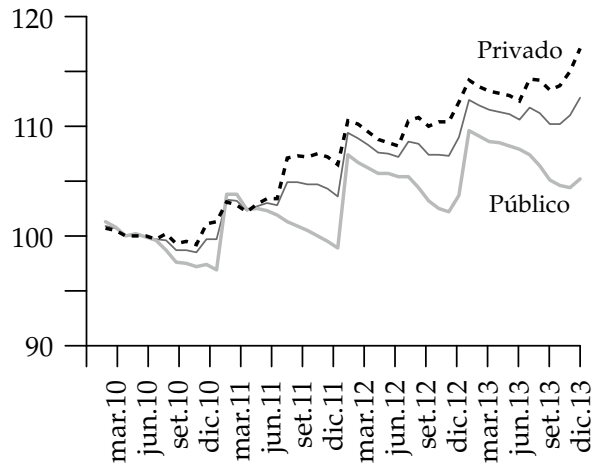

Inflación

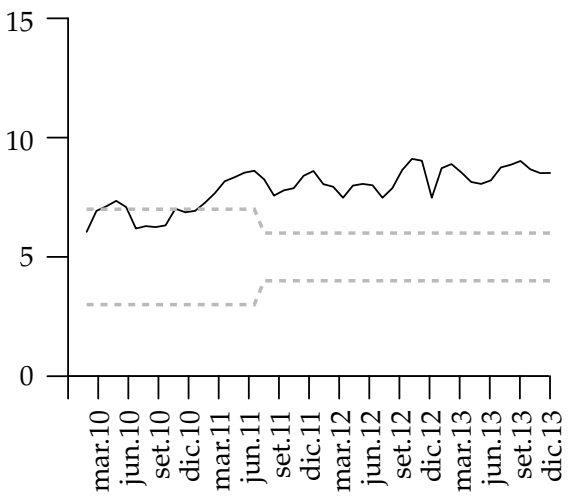

Desempleo

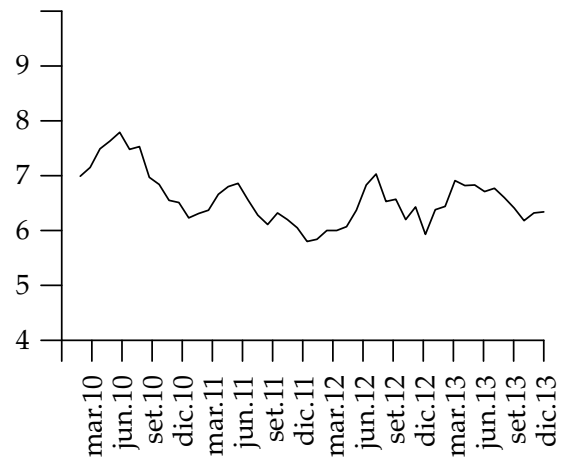

Fuente: elaborado a base de datos del INE y BCU.

lar a lo que se conoce como "inflación subyacente", 1 lo que sugiere importantes límites en la efectividad de las medidas de control de precios asociadas a las tarifas de servicios.

En tercer lugar, los niveles de coordinación económica instrumentados mediante la negociación colectiva (tripartita) han permitido un aumento moderado del salario real. La evolución del salario real durante 2013 se inicia con un crecimiento importante del poder de compra de los hogares, luego de la recuperación salarial impuesta por los laudos de la negociación tripartita de 2012. Sin embargo, el incremento de los precios evitó el subsecuente crecimiento del salario real en el sector privado y deterioró el nivel del salario real en el sector público. Aun así, los niveles salariales aumentaron $3,3 \%$ durante 2013 . 
Unido a lo anterior, una de las tensiones más relevantes en la política económica refiere a los efectos de la política salarial sobre la inflación. Debido a que la productividad de los factores está cerca de su techo, el incremento de los salarios por encima del incremento en la productividad ha sido señalado como un factor importante para explicar la incapacidad del gobierno para reducir la inflación. Sin embargo, la política de negociación salarial es una parte fundamental del proyecto político de la izquierda que ha permitido distribuir el ingreso hacia los trabajadores. Más aún, luego de la pérdida de salario real ocurrida durante la crisis del 2002-2003, el salario ha crecido de manera sostenida, tanto en términos nominales como reales, permitiendo a los trabajadores incrementar su poder adquisitivo. En este sentido, el salario ha tenido un rol dinamizador de la demanda interna, cada vez más importante como componente de la explicación del crecimiento económico.

En cuarto lugar, el déficit del sector público alcanzó al 2,3\% del PIB. Este resultado supera las previsiones del gobierno realizadas en 2012 que estimaban cerrar el año con un déficit del 2,1\% del PIB. El resultado fiscal de 2012 ya había marcado el peor desempeño fiscal desde 2002, con un déficit que alcanzó 2,8\% del PIB (BCU). Uno los aspectos más notables del gasto público en Uruguay tiene que ver con su alto nivel de rigidez y crecimiento incremental poco reversible. Esto hace que en contextos de desaceleración del crecimiento económico la capacidad del gobierno para controlar los niveles de déficit fiscal es reducida.

Finalmente, desde que Uruguay abandonó el sistema cambiario de bandas y pasó a un régimen de flotación libre, la depreciación del peso trajo un incremento importante de la competitividad de los productos uruguayos en el mercado internacional. Una vez que el peso se comienza a revaluar a partir de 2004, Uruguay comienza a perder progresivamente su competitividad exportadora. El tipo de cambio real de la economía uruguaya respecto de sus principales socios comerciales cayó sustancialmente entre 2009 y 2010. En 2013 se da un preocupante proceso de pérdida de competitividad de 7,6\% que tuvo lugar en los primeros ocho meses del año respecto de igual período del año anterior. Esa tendencia se revierte en los últimos cuatro meses y el resultado es de un incremento porcentual (depreciación real) de 5,2\% respecto del de diciembre de $2012 .^{2}$ Lo que resulta relevante para comprender la tendencia de la inserción de Uruguay en el mercado internacional es que en el mediano plazo la mayor caída de la competitividad se registra en las exportaciones a destinos extrarregionales.

\section{EL PODER EJECUTIVO Y LA AGENDA DE GOBIERNO}

Desde la reinstauración democrática en 1985, solo los gobiernos del Frente Amplio han gozado de mayorías de partido para gobernar, mientras los gobiernos liderados por el Partido Colorado o el Partido Nacional han sido predominantemente gobiernos de coalición. La diferencia entre uno y otro tipo de gobierno tiene implicaciones importan- 
tes para la estabilidad de los gabinetes y la gestión del gobierno. Formar un gobierno en condición de partido mayoritario implica (en el peor de los casos) una negociación interna dentro del partido ganador de la elección. Formar un gobierno de coalición implica una compleja ingeniería programática, además de los arreglos y negociaciones dentro de los partidos que deciden entrar en una coalición. Por este motivo, los gobiernos de partido en mayoría tienen una mayor estabilidad del gabinete, comparado con los gobiernos de coalición. Una rápida observación a la evidencia uruguaya desde la restauración democrática hace evidente esta aseveración.

Un aspecto crítico en la formación del gobierno está dado por la distribución de cargos en el gabinete, en función del tamaño de los sectores que forman parte del partido o coalición de gobierno. Precisamente, el Índice de Congruencia Parlamentaria permite calcular la proporcionalidad entre el porcentaje de carteras asignadas entre sectores y el porcentaje de bancas que tiene cada sector en el Legislativo (Amorim Neto, 1998). De acuerdo con el Cuadro 1, la integración de los gabinetes es más inestable bajo distintas modalidades de gobierno de coalición que en casos de partido mayoritario. Una simple comparación de los gobiernos anteriores a la llegada del Frente Amplio en 2005 muestra claramente que la rotación de los elencos partidarios en el gabinete era mayor que en el periodo iniciado con Vázquez y luego seguido por el presidente Mujica (Buquet y Luján, 2013).

El Cuadro 1 no solo muestra el esfuerzo del Frente Amplio por preservar la proporcionalidad entre votos y puestos en el gabinete, sino que además muestra que el Frente Amplio no tuvo fisuras durante los dos periodos de gobierno. Durante el periodo del presidente Vázquez, el gabinete fue integrado por los líderes de las fracciones del Frente Amplio, poniéndose un particular cuidado en el balance entre fracciones a nivel de ministerios, subsecretarías y otros cargos de particular confianza. Lo mismo puede decirse del periodo del presidente Mujica, porque no han existido cambios en la distribución

Cuadro 1. Estructura del gobierno y gabinetes ministeriales: Uruguay 1985-2013

\begin{tabular}{lccccccc}
\hline Presidentes & Período & Tipo de gabinete & Ministros & $\begin{array}{c}\text { Partido de } \\
\text { Presidente }\end{array}$ & $\begin{array}{c}\text { Partidos } \\
\text { Socios }\end{array}$ & Independientes & ICFG \\
\hline Sanguinetti I & 1985-90 & Partido Minoritario & 11 & 8 & 0 & 3 & 0.899 \\
Lacalle & $1990-92$ & Coalición Mayoritaria & 12 & 8 & 4 & 0 & 0.818 \\
- & $1992-93$ & Coalición Minoritaria & 12 & 10 & 2 & 0 & 0.742 \\
- & $1993-95$ & Coalición Minoritaria & 12 & 10 & 2 & 0 & 0.754 \\
Sanguinetti II & $1995-00$ & Coalición Mayoritaria & 12 & 7 & 4 & 1 & 0.813 \\
Batlle & 2000-02 & Coalición Mayoritaria & 13 & 8 & 5 & 0 & 0.91 \\
- & $2002-05$ & Partido Minoritario & 13 & 11 & 0 & 2 & 0.839 \\
Vázquez & $2005-10$ & Partido Mayoritario & 13 & 12 & 0 & 1 & 0.801 \\
Mujica & $2010-13$ & Partido Mayoritario & 13 & 12 & 0 & 1 & 0.835 \\
\hline
\end{tabular}

Fuente: Chasquetti y Gueddes, 2013. 
proporcional de cargos al final del cuarto año de gobierno. Sin embargo, llegado el cuarto año de gobierno de Vázquez, todos los ministros líderes de fracción decidieron volcarse a la arena política en el Parlamento, a los efectos de enfrentar la competencia interna (dentro del Frente Amplio) y luego la elección nacional de octubre y noviembre de 2009. Estos cargos fueron reemplazados por miembros de las mismas fracciones de los ministros salientes y por tanto no existieron cambios en el índice dentro del periodo.

Durante 2013 hubo solo dos cambios en el gabinete. El primero de ellos fue el ministro de Salud Pública Jorge Venegas, tras la denuncia del senador Amorin Batlle del Partido Colorado. Venegas se encontraba en irregularidad en el ejercicio de su cargo y presentó su renuncia, siendo sustituido el 25 de febrero por Cristina Muñiz, también perteneciente al Partido Comunista (Frente Amplio). El segundo cambio ocurrido en el gabinete se produjo en diciembre de 2013, tras la salida del ministro de Economía (Fernando Lorenzo) y su sustitución por el Presidente del Banco Central del Uruguay (Mario Bergara), ambos pertenecientes al Frente Líber Seregni (liderado por el vicepresidente Danilo Astori). Nótese que el índice de congruencia del Cuadro 1 no varía durante el periodo de Mujica, ya que la simetría entre votos a fracciones y puestos en el gabinete permaneció incambiada.

Por el contrario, los cambios verificados a nivel de las subsecretarías y otros cargos dentro del Poder Ejecutivo sí obedecieron a motivaciones políticas dentro del partido de gobierno. En este nivel hubo varios cambios durante 2013. El más importante a consignar es la salida del viceministro de economía Luis Porto para ocupar el cargo de viceministro en el ministerio de relaciones exteriores. El origen de este cambio es complejo e involucra fuertemente a la interna del partido de gobierno. Las elecciones para la presidencia del Frente Amplio a padrón abierto en 2012 dejaron a la senadora Mónica Xavier como presidenta de la coalición de izquierda, luego de una intensa competencia entre cuatro candidatos con distinta orientación ideológica (Monestier, 2013). Su llegada a la presidencia vino acompañada de algunos conflictos entre los sectores moderados que le habían dado su apoyo con aquellos del ala izquierda que competían con su candidatura. Una vez elegida, el Partido Comunista y el MPP orientaron sus críticas hacia la incompatibilidad entre los cargos de presidenta del Frente Amplio y su banca en el Senado representando al Partido Socialista. Luego de un debate cruzado de varias semanas, Mónica Xavier optó por renunciar a su banca en el Senado, dando paso a su suplente, el vicecanciller Roberto Conde. La vacante de Conde en el Ministerio de Relaciones Exteriores motivó al Presidente a nombrar a Porto como vicecanciller, dejando a su vez vacante la subsecretaría de Economía y Finanzas.

Esta última vacante suscitó un conflicto aun mayor dentro del Frente Amplio. La salida del subsecretario Porto en Economía dio pie a que el Presidente designara al socialista Alejandro Antonelli en forma inconsulta con su propio partido y el Frente Líber Seregni. Antonelli, un miembro del sector más conservador y predominante en la interna del partido socialista no solo no contaba con credenciales técnicas de renombre dentro y fuera de la coalición de izquierda, sino que además no contaba con la aceptación del ministro Lorenzo y los sectores moderados del Partido Socialista. La designación de Antonelli produjo un malestar importante entre algunos sectores del Frente Amplio, 
porque fue vista como un mecanismo de control de la política económica (en manos del Frente Líber Seregni), además del contrapeso ya existente en la Oficina de Planeamiento y Presupuesto (en manos del propio Presidente).

El conflicto dentro del gabinete llegó a su máxima expresión a inicios de febrero de 2013, luego de una serie de designaciones derivadas de la elección interna por la presidencia del Frente Amplio. Durante varias semanas el Frente Amplio y en particular las cabezas visibles del conflicto intercambiaron opiniones y polemizaron en los medios a raíz de las decisiones del Presidente, al punto en que el Consejo de Ministros no sesionó durante más de dos meses, luego de que estallara el conflicto por las designaciones (el 20 de febrero). En suma, es importante observar que los partidos en su mayoría no están exentos de conflictos internos. Los partidos se componen de grupos, tendencias, y en el último de los casos fracciones altamente visibles con liderazgos estables en el tiempo. El Frente Amplio está integrado por fracciones con una distancia ideológica importante, lo que se ha visto reflejado en altos niveles de competencia por el voto y conflictos de distinta índole, fundamentalmente desde que accedió al gobierno en 2005. La proximidad de los procesos electorales no hace otra cosa que volver más visibles estos conflictos, como ha ocurrido en 2013.

\section{EL GOBIERNO Y LA GESTIÓN DE LA AGENDA}

El gobierno de José Mujica enfrentó algunas dificultades en la aprobación de legislación durante los primeros dos años de su gestión (2010 y 2011), seguidos por niveles muy satisfactorios en la aprobación de legislación promovida por el Poder Ejecutivo en 2012 y 2013 (Chasquetti y Guedes, 2013). A pesar de la estabilidad del gabinete y el éxito del gobierno en la arena legislativa donde tiene mayoría, el gobierno ha mostrado éxitos y algunas dificultades, derivadas del desempeño en ciertas áreas de política pública de gran impacto a nivel de la opinión pública.

Por un lado, el gobierno ha logrado aprobar legislación que expande la provisión de derechos sociales. Esta agenda está compuesta por legislación que el Frente Amplio ha promovido tradicionalmente, fuera y dentro del gobierno. Un ejemplo importante de este tipo de políticas fue aprobado en 2013 , con la nueva ley de subsidios por maternidad y paternidad para trabajadores de la actividad privada, aprobada por unanimidad el 24 de octubre en el Senado. Otro ejemplo notorio es la inclusión de nuevos colectivos en el sistema nacional de salud, lo que expande notoriamente el universo de potenciales afiliados al sistema mutual de salud. Esta es la agenda clásica de un gobierno de izquierda.

Por otro lado, la nueva agenda de asuntos promovidos por el Frente Amplio pretende satisfacer al electorado de izquierda libertaria, que tradicionalmente ha caracterizado a una parte de los votantes del Frente Amplio. Esta agenda ha encontrado algunas voces opositoras dentro del propio partido de gobierno a la hora de promover legislación vinculada al aborto, la legalización de la producción y comercialización de marihuana o la aplicación de los derechos de matrimonio a parejas de un mismo sexo. Otras áreas de expansión de derechos, ciertamente menos polémicas que las anteriores, fueron la apro- 
bación de la ley de reproducción asistida (que incluye dentro del sistema de salud el tratamiento a las parejas imposibilitadas de tener hijos en forma natural), la ampliación de la licencia laboral maternal y paternal, así como la reglamentación de la eutanasia en la llamada ley del "buen morir". El Frente Amplio ha logrado aprobar de forma exitosa esta nueva generación de derechos típicamente asociados a la izquierda libertaria.

Este cuerpo de legislación tiene como punta del iceberg al proyecto de legalización del aborto (o interrupción voluntaria del embarazo), sancionado el 22 de octubre de 2012, pero finalmente zanjado política y legalmente en 2013 con el fracaso de la interposición de firmas para promover un referéndum derogatorio de la ley (18.987). Durante el gobierno del presidente Vázquez, un proyecto de similares características había sucumbido en el legislativo, luego de ser aprobado por ambas Cámaras pero posteriormente vetado por el Presidente. Bajo el gobierno de Mujica el Parlamento finalmente dio aprobación al proyecto que si bien levantaba algunas objeciones minoritarias dentro del Frente Amplio y fundamentalmente en la oposición, existía un consenso amplio, acompañado de un gran nivel de presión de grupos de activistas marcadamente vinculados a la izquierda.

Un segundo proyecto en la agenda de derechos de corte libertario durante 2013 está dado por la aprobación de los derechos para la contracción de matrimonio igualitario o para parejas de un mismo sexo. Este proyecto también encontró voces contrarias, pero se concentraron primordialmente en una minoría de la oposición colorada y fundamentalmente dentro del Partido Nacional. Al igual que en el aborto, el objetivo del sistema político no solo ha estado orientado a otorgar un derecho a un grupo considerable de personas con determinada orientación sexual, sino que además la legislación viene a resolver algunos problemas o dilemas legales de miles de parejas de un mismo sexo. A diferencia del aborto, no obstante, la aprobación de la ley en 2013 estuvo acompañada de un mayor nivel de consenso en el sistema político y la opinión pública.

Por último, la agenda libertaria del gobierno recaló en una de las áreas de política pública más polémicas a nivel doméstico e internacional: la regulación del consumo, producción, y comercialización de la Cannabis. ${ }^{3}$ Este paso del gobierno, a diferencia del aborto y los derechos matrimoniales en parejas de un mismo sexo, contó con una acción proactiva del presidente Mujica, quien salió públicamente a explicitar los beneficios de un proyecto que dejaba dudas dentro del Frente Amplio y entre los partidos de oposición. Nótese que este tema ha circunvalado la arena legislativa en varias oportunidades y venía de un fracaso en la legislatura anterior. Fruto de su ajustada mayoría absoluta en el legislativo, el Frente Amplio tuvo que zurcir finamente algunos votos no dispuestos a apoyar el proyecto del Ejecutivo, finalmente aprobado el 31 de diciembre de 2013, con los votos de toda la bancada del Frente Amplio.

Es importante notar que esta agenda libertaria no forma parte del programa actual de gobierno del Frente Amplio y tampoco ha sido parte de las negociaciones con la oposi-

La ley establece tres formas en las que un consumidor puede obtener marihuana: el autocultivo, por el cual cada persona podrá tener hasta seis plantas o producción de hasta 480 gramos; b) clubes de membresía, los cuales podrán tener de 15 a 45 socios y un máximo de 99 plantas; c) adquirir la producción estatal. 
ción al inicio del gobierno de Mujica. Sin embargo, estos tres asuntos de política pública no son menores. Pone al país a la vanguardia en materia de ciertos derechos, le permite al Frente Amplio conservar un electorado de izquierda con cierto nivel de activismo, mejorándole al Presidente una imagen que en otras áreas de política pública se ha visto deteriorada debido a los resultados de la gestión. Desde este punto de vista, 2013 fue un éxito para el gobierno.

\section{Algunas sombras}

Las dificultades en la gestión y algunos fracasos en áreas importantes de política pública se han tornado altamente visibles con el avance del periodo de gobierno y el tratamiento que la oposición ha hecho de estos y otros asuntos de cara a la competencia electoral en 2014. En particular, vale la pena resaltar tres áreas en las cuales el gobierno no ha logrado los resultados de gestión que esperaba en 2013. Estas son áreas que la opinión pública ha identificado en forma estable como los principales problemas que enfrenta el país. ${ }^{4}$ En primer lugar, la seguridad pública sigue ocupando el principal lugar en la agenda de preocupaciones, tanto del gobierno como de la opinión pública. De acuerdo con cifras oficiales, el número de robos con violencia -el delito cometido con mayor frecuencia en Uruguay- creció en 2013 8,1\%, a igual periodo de 2012. Este dato se suma a las críticas sostenidas en forma sistemática por parte de organismos nacionales e internacionales sobre la violación de derechos humanos en el sistema cárceles.

Si bien han existido algunos avances en la seguridad pública y la política carcelaria, el ritmo no ha acompasado las necesidades y el gobierno todavía se ve sometido a críticas y evaluaciones negativas. Con todo, ni los resultados de la gestión ni la evaluación de la opinión pública han puesto en jaque al ministro a cargo de esta cartera (Bonomi), pero tampoco al gobierno en un año preelectoral y en vísperas de un plebiscito sobre la baja en la edad de imputabilidad penal (de 18 a 16 años). Este plebiscito fue impulsado por uno de los líderes colorados de la oposición (Pedro Bordaberry) mediante la recolección de firmas, en un proceso en el que se sumó parcialmente el Partido Nacional y que tendrá en la próxima campaña un lugar central, debido al carácter concomitante con la realización de las elecciones nacionales.

En segundo lugar, cabe destacar los problemas que el gobierno ha tenido en el área educativa, donde los resultados de la gestión en 2013 no muestran mejoría, a pesar de los niveles de gasto en este sector. En 2013 el gobierno debió divulgar cifras muy negativas de repetición y culminación del ciclo básico de educación secundaria, que ponen al país entre los de peor desempeño de América Latina. Buscando revertir los resultados, el Presidente ya había removido en sus cargos a los presidentes del Consejo Directivo Central (CODICEN) y la Administración Nacional de Educación Pública (ANEP) hacia finales de 2012, pero las críticas no cesaron durante 2013, forzando al gobierno a hacer

$4 \quad$ Ver: “El principal problema del país es la inseguridad”, en http://www.uypress.net/uc_47567_1.html 
una defensa en la que el propio candidato presidencial del Frente Amplio (y expresidente) Tabaré Vázquez ha retado a la oposición a debatir sobre los resultados en el área educativa. El único éxito visible en esta área no tiene todavía resultados de gestión, luego de la aprobación de la Universidad Tecnológica (UTEC) el 20 de diciembre de 2012.

La tercer área crítica para el gobierno es la vivienda. Esta política no era parte de los acuerdos interpartidarios propiciados por Mujica en su primer año de gobierno, como fue la educación y la seguridad pública. Sin embargo, no parece mostrar mayor éxito al cabo del cuarto año de gobierno. Bajo la égida de la Presidencia de la República, el Presidente creó el Plan Juntos para dar solución al problema de la vivienda en sectores de bajos ingresos. El plan ha tenido como objetivo proveer vivienda y regularizar la expansión de viviendas en asentamientos irregulares de zonas urbanas. Sin embargo, no solo no ha contado con escasos fondos públicos sino que además no ha tenido la acogida que el propio Presidente esperaba que tuviera su iniciativa entre donadores públicos y privados. A falta de resultados positivos, reflejados en los cambios ocurridos en el gabinete con la sustitución de la ministra Muslera en 2012 y otros cargos relevantes de la gestión de la política de vivienda (en el Banco Hipotecario y la Agencia Nacional de Vivienda), esta área de política pública emerge en 2013 (y de cara a 2014) como un asunto importante de la campaña, con propuestas maximalistas de la oposición, tendientes a erradicar los asentamientos irregulares.

En el marco de estos resultados de la gestión, otros problemas han ocupado el interés del gobierno y la oposición durante 2013, en asuntos que ya despuntan como ejes de la campaña electoral de 2014. El primero de ellos es el Pluna-gate: un asunto derivado de la reestructuración de la empresa estatal de aviación civil en 2006, gerenciada por un consorcio público-privado entre el Estado y la empresa Leadgate. La sociedad fracasó en 2012 y el gobierno inició el proceso de quiebra y liquidación de la empresa. Este proceso iniciado hacia fines de 2012 ocupó la atención del sistema político durante todo el 2013, transformándose en el principal interés de la oposición en el Parlamento. Este complejo asunto, que tiene como elemento más problemático el otorgamiento irregular de un aval financiero al empresario José López Mena por parte del Banco de la República (con el aval del Ministerio de Economía) para la compra de los aviones de la liquidación de Pluna, derivando en los tribunales con la acusación al ministro y el presidente del Banco por abuso de funciones. En diciembre de 2013 el ministro renunció a su cargo tras ser procesado sin prisión en esta causa.

El segundo asunto que ha acaparado la atención del gobierno durante 2013 es el Impuesto a las Contribuciones Inmobiliarias Rurales (ICIR). Este impuesto orientado a recuadrar fondos públicos para financiar infraestructura o caminos rurales levantó el rechazo de las gremiales agrarias y la oposición al gobierno, dándole curso a una demanda legal por inconstitucionalidad de la ley (18.876). Precisamente, en 2013 la Suprema Corte de Justicia declaró inconstitucional la ley del gobierno, observando una política que el Presidente (no así el ministro de economía) había impulsado desde el inicio de su mandato. Este impuesto, así como la ley de minería de gran porte aprobada en 2013 (Ley 19.126), el conflicto con Argentina por la ampliación de la cuota de producción de la 
empresa papelera UPM (localizada en el río Uruguay) y consiguiente sanción indirecta de Argentina a la actividad de los puertos uruguayos y otros aspectos de la política exterior (como la relación bilateral con Venezuela), han mantenido la atención del gobierno y la oposición durante 2013.

Desde el inicio del gobierno del presidente Tabaré Vázquez la política económica ha estado en manos de los sectores moderados del Frente Amplio. Durante la construcción del actual gabinete el presidente Mujica dio su respaldo al vicepresidente Astori y su gestión de la economía durante el gobierno de Vázquez, designando al mismo equipo al frente del Ministerio de Economía (Fernando Lorenzo), el Banco Central (Mario Bergara) y el Banco de la República (Roberto Calloia). Sin embargo, buscando darle balance a la orientación de la política económica, el presidente Mujica designó a dos economistas de su confianza personal al frente de la Oficina de Planeamiento y Presupuesto (OPP). Este diseño del gabinete obedece largamente a la oposición entre los sectores más radicales (MPP y Comunistas) con los sectores ideológicamente moderados del Frente Amplio (Socialistas y Frente Líber Seregni), a cargo de la política económica (Monestier, 2013).

Un ejemplo de la compleja política interna del Frente Amplio se halla en el ICIR. Diseñado en la OPP y promovido vigorosamente por el presidente Mujica, el ICIR contó desde 2010 con la oposición del Ministro de Economía (Fernando Lorenzo). La negociación a la interna del Frente Amplio por la imposición del impuesto dio lugar a una dura batalla por lograr un punto intermedio entre las preferencias de OPP y el Ministerio de Economía. Estaba en juego la política de impuestos del gobierno y la reputación o credibilidad del propio ministro Lorenzo, quien había sostenido que la política impositiva no habría de sufrir modificaciones durante este período. En este contexto, el vicepresidente y aval político del ministro de Economía había sostenido durante las negociaciones internas, que el impuesto era inconstitucional y que la aprobación de la ley podría hacer enfrentar una futura derrota legal al gobierno. Luego de una ardua discusión interna, acompañada de amenazas de renuncia por parte del ministro Lorenzo, el impuesto fue negociado y aprobado en el legislativo (Ley 18.876).

El gobierno también se enfrentó a las demandas de movimientos sociales como los ambientalistas movilizados contra la aprobada ley que regula la minería de gran porte. Los críticos de la megaminería a cielo abierto denuncian que la legislación fue formulada a medida de la empresa Aratirí. También hay actores que denuncian la eventual ineficacia de los impuestos creados para recaudar ingresos provenientes de la megaminería. Aún así, el gobierno siguió adelante con el proyecto y aprobó una ley que abre paso a la expansión de la actividad minera en el país.

En el plano internacional los conflictos con Argentina han continuado la tónica de la última década. La empresa fábrica de celulosa UPM (ex-Botnia) ejerció presión con el fin de aumentar su actividad, lo que llevó al gobierno de Mujica a atravesar una nueva crisis en las relaciones con Argentina. Luego que Mujica anuncia la autorización a UPM a aumentar su producción, el gobierno de Argentina responde con anunciar un nuevo recurso a la Corte Internacional de La Haya para dirimir el conflicto. Posteriormente, el gobierno de Cristina Fernández prohíbe que las exportaciones argentinas hagan transbordos 
en puertos uruguayos. No obstante, los líderes políticos de todos los partidos han continuado con su tónica de apoyo al gobierno cuando se trata del conflicto con Argentina.

Durante 2013, el Presidente ha tomado un perfil distinto al de sus primeros tres años (Moraes et al., 2012). La gestión del liderazgo presidencial pasó de tener un carácter proactivo pero vacilante en varias áreas de política pública como los impuestos, la minería de gran porte y la vivienda, para tomar un perfil bajo y búsqueda de acuerdos a la interna del partido. Esta tendencia se vio interrumpida por la estrepitosa designación del viceministro de Economía (Alejandro Antonelli), pero ha sido la tónica general de la segunda parte del mandato del presidente. Mientras que en los primeros años hizo un esfuerzo importante por salir a los medios y la opinión pública para explicitar sus preferencias de política pública en general, durante 2013 se mostró más selectivo en las políticas donde invertir su capital político (Chasquetti y Guedes, 2013).

\section{EL PODER LEGISLATIVO}

Todo Legislativo en un sistema presidencialista cumple con dos funciones fundamentales. Por un lado es colegislador junto al Poder Ejecutivo. Esto es, no hay legislación si estos dos poderes no se ponen de acuerdo sobre cada proyecto de ley presentado en el Parlamento. Por otro lado, el Legislativo cumple con una tarea fundamental en la mecánica del sistema republicano, al tener que controlar las actividades del Poder Ejecutivo. Sin control del Legislativo, no hay monitoreo en la gestión del gobierno, debilitándose los mecanismos de rendición de cuentas a los partidos de oposición y a los ciudadanos en general. Precisamente, la producción legislativa y el contralor del Ejecutivo son las dos funciones principales que importa consignar a la hora de observar el funcionamiento del sistema político en 2013.

En primer lugar, la producción legislativa durante el periodo de gobierno del presidente Mujica ha mostrado un patrón similar (aunque no idéntico) al observado en los periodos posteriores a la reinstauración democrática: neto predominio del Ejecutivo sobre el Legislativo y un patrón de creciente eficiencia en el pasaje de legislación con origen en el Ejecutivo. Estas dos constantes han tenido lugar en forma sistemática desde 1985, aunque el nivel de predomino del Ejecutivo depende en gran medida de las mayorías con las que ha contado cada Presidente en el Parlamento. Precisamente, el presidente Mujica cuenta con una mayoría absoluta de votos en el Legislativo, que siempre y cuando se comporte en forma disciplinada, le permite aprobar la legislación propuesta por el Ejecutivo. Sin embargo, a diferencia del gobierno de Vázquez (que detentaba una mayoría absoluta apenas más holgada) y cada uno de los gobiernos desde la reinstauración, Mujica comenzó su mandato con tasas moderadas de aprobación de legislación en el entorno del 35\% en 2010, para mejorarlos ostensiblemente en 2011 y 2012 (con 56\%, y $68 \%$, respectivamente) y más recientemente $68 \%$ en 2013 (Chasquetti y Guedes, 2013).

De acuerdo con el Cuadro 2 el cuatrienio móvil 2010-2013 muestra una tasa idéntica a la del periodo 2005-2008, perteneciente al gobierno del presidente Vázquez. Ambos gobiernos han operado bajo la lógica de un partido en mayoría absoluta en el Legislativo, 
Cuadro 2. Tasa de eficacia de los poderes: trienios móviles 1985-2013 (entre paréntesis leyes/proyectos)

\begin{tabular}{|c|c|c|c|c|c|c|}
\hline \multirow{2}{*}{ Iniciativa } & Sanguinetti & Lacalle & Sanguinetti & Batlle & Vázquez & Mujica \\
\hline & $1985-88$ & $1990-93$ & $1995-98$ & $2000-03$ & $2005-08$ & $2010-13$ \\
\hline \multirow{2}{*}{ Poder Ejecutivo } & $48 \%$ & $50 \%$ & $51 \%$ & $58 \%$ & $67 \%$ & $67 \%$ \\
\hline & $(141 / 296)$ & $(203 / 406)$ & $(193 / 375)$ & $297 / 510)$ & $(317 / 472)$ & $(352 / 523)$ \\
\hline \multirow{2}{*}{ Poder Legislativo ${ }^{* *}$} & $12 \%$ & $13 \%$ & $17 \%$ & $18 \%$ & $22 \%$ & $18 \%$ \\
\hline & $(94 / 793)$ & $(87 / 683)$ & $(105 / 624)$ & $(148 / 809)$ & $(151 / 702)$ & $(106 / 599)$ \\
\hline \multirow[t]{2}{*}{ Total } & $22 \%$ & $27 \%$ & $30 \%$ & $34 \%$ & $40 \%$ & $41 \%$ \\
\hline & $(235 / 1090)$ & $(290 / 1089)$ & $(298 / 1001)$ & $(445 / 1319)$ & $(469 / 1175)$ & $(458 / 1122)$ \\
\hline
\end{tabular}

Notas: El número de leyes/ proyectos se especifican entre paréntesis. ${ }^{*}$ Entre el 1 de marzo del primer año y el 31 de julio del cuarto año. ${ }^{* *}$ En las legislaturas $45^{\mathrm{a}}, 46^{\mathrm{a}}$ y $47^{\mathrm{a}}$ se incluyen proyectos presentados por organismos como el Tribunal de lo Contencioso Administrativo, el Tribunal de Cuentas, la Corte Electoral y la Suprema Corte de Justicia (2, 3 y 2, respectivamente).

Fuente: Chasquetti y Guedes 2013, en base a datos del Sistema de Información Parlamentaria.

lo que se ha visto reflejado en una aprobación de dos terceras partes de la legislación propuesta por el Poder Ejecutivo (67\%). En este contexto, las tasas de aprobación en proyectos presentados por el Legislativo son ostensiblemente menores a las del Ejecutivo, pero son relativamente estables desde el segundo mandato del presidente Sanguinetti (1995-2000).

Desde un punto de vista formal, el gobierno liderado por Mujica muestra un éxito evidente en su estrategia de aprobación de legislación. Sin embargo, desde un punto de vista sustantivo, el Legislativo uruguayo no es un agente inocuo en el sistema político. Como sostiene Chasquetti (2014), el Parlamento no es un "sello de goma" para las iniciativas del Ejecutivo, aun en condiciones de partido mayoritario. Esta configuración queda demostrada en al menos dos indicadores que revelan la forma en que aun un partido mayoritario con la capacidad de cartelizar la producción legislativa, también es capaz de incidir en la legislación mediante la modificación de las iniciativas del Ejecutivo. Esto permite un ajuste entre las preferencias del Ejecutivo y las preferencias del legislador mediano del partido mayoritario, dando aprobación a la agenda del Ejecutivo pero con control del Legislativo.

Obsérvese que la duración promedio para aprobar una ley en el actual periodo de gobierno ha sido de 270 días, o 233 y 394 días para proyectos del Ejecutivo y el Legislativo, respectivamente (Chasquetti, 2014). Con estos tiempos de tramitación, el Ejecutivo tiende a lograr lo que quiere, pero a un cierto costo de negociación (dentro y muchas veces fuera de la bancada del partido de gobierno). De hecho, durante el actual periodo de gobierno el Parlamento hizo algún tipo de modificaciones en alrededor de un tercio de la legislación con iniciativa en el Ejecutivo (31\%). Naturalmente, también los proyectos presentados por los legisladores son modificados en el proceso legislativo 
y de hecho sufren más modificaciones que los proyectos con iniciativa en el Ejecutivo. Pero esta no es la agenda del gobierno, sino del partido de gobierno en el Legislativo o los partidos de oposición.

A inicios de 2013 el gobierno no solo propuso una agenda de iniciativas legislativas a ser tratadas en el Parlamento, sino que además recibía el rezago en la aprobación de legislación de años anteriores. Como muestra el Cuadro 3, al menos dos proyectos importantes impulsados por el Presidente a inicios de 2013 fueron tramitados dentro del promedio de días en proyectos del Ejecutivo. Los restantes proyectos prioritarios para la agenda del gobierno en 2013 son rezagos de legislación iniciada en años anteriores. En suma, el gobierno tiende a lograr sus objetivos en la aprobación de políticas, pero debe ser paciente con el procesamiento de su agenda. Esto no es otra cosa que un dato positivo sobre el funcionamiento de la democracia y en particular sobre las instituciones políticas uruguayas.

En segundo lugar, otro indicador fundamental del funcionamiento del sistema político se centra en las actividades de control del gobierno (Chasquetti y Guedes, 2013; Chasquetti, 2014). También aquí la actividad es intensa, ya que pone al descubierto la interacción del gobierno con los partidos de la oposición en el Parlamento. Desde un punto de vista institucional, el eje del control sobre la gestión del gobierno tiene lugar en el Parlamento, para lo cual la oposición y en particular sus miembros tomados individualmente pueden hacer uso de algunos dispositivos como los llamados a sala de los ministros y los pedidos de informes al Ejecutivo. Estos dos instrumentos son de uso permanente y en numerosas oportunidades los propios legisladores de la bancada de gobierno contribuyen con su voto para llamar a los ministros en ré-

Cuadro 3. Agenda prioritaria para el gobierno

\begin{tabular}{lccc}
\hline \multicolumn{1}{c}{ Asunto } & $\begin{array}{c}\text { ¿Proyecto } \\
\text { presentado? }\end{array}$ & $\begin{array}{c}\text { Fecha de } \\
\text { presentación }\end{array}$ & $\begin{array}{c}\text { ¿Proyecto } \\
\text { aprobado? }\end{array}$ \\
\hline Creación del Instituto Nacional de Meteorología & Sí & $13 / 3 / 2013$ & $16 / 10 / 2013$ \\
Readecuaciones orgánicas en las FF.AA. & Sí & $23 / 1 / 2013$ & $9 / 10 / 2013$ \\
Minería de gran porte & Sí & $16 / 12 / 2012$ & $22 / 9 / 2013$ \\
Reformulación del estatuto del funcionario público & Sí & $19 / 10 / 2012$ & $20 / 8 / 2013$ \\
Ley de regulación de la marihuana & Sí & $8 / 8 / 2012$ & $10 / 12 / 2013$ \\
Instituto Nacional de Formación (IUDE) & Sí & $15 / 5 / 2012$ & No \\
Ley orgánica del sistema penitenciario & Sí & $12 / 8 / 2011$ & No \\
Ley de residencias médicas. & Sí & $23 / 11 / 2011$ & No \\
Reformas en el código procesal penal & Sí & $8 / 12 / 2010$ & No \\
Pasaje Justicia Militar a Justicia Civil & No & - & - \\
Ley de sanciones a la competencia desleal & No & - & - \\
Actualización del estatuto del trabajador rural & No & - & - \\
\hline
\end{tabular}

Fuente: Chasquetti, 2014. 
gimen de comisión permanente o piden informes al Ejecutivo al igual que cualquier otro miembro de la oposición.

El Cuadro 4 muestra los asuntos que han acaparado la atención de la oposición durante 2012 y 2013. A diferencia de los primeros años de gobierno, donde la oposición mantenía cierta dispersión temática en el control del Ejecutivo, 2013 muestra una clara concentración del interés en el Pluna-gate. Una vez que entró en quiebra la empresa de bandera uruguaya (hacia fines de 2102), la liquidación de la empresa ha hecho pasar al gobierno por un proceso tormentoso del cual no logra salir y probablemente no lo logre, en fun-

Cuadro 4. Llamados a sala y en régimen de comisión general. Uruguay 2012-13*

\begin{tabular}{|c|c|c|c|c|c|c|}
\hline Ingreso & Tipo & Cámara & Iniciativa & Ministerio & Ministro & Motivo \\
\hline $16-07-13$ & LS & CSS & PN-PC & BROU & Caloia & Pluna. Pago aval \\
\hline $16-07-13$ & LS & CSS & PN-PC & MTOP & Pintado & Pluna. Pago aval \\
\hline $16-07-13$ & LS & CSS & PN-PC & MEF & Lorenzo & Pluna. Pago aval \\
\hline $10-07-13$ & CG & CSS & PN-PC & MTOP & Pintado & $\begin{array}{l}\text { Pluna S.A. Información acuerdo y } \\
\text { pago aval BROU. }\end{array}$ \\
\hline $10-07-13$ & CG & CSS & PN-PC & BROU & Caloia & $\begin{array}{l}\text { Pluna S.A. Información acuerdo y } \\
\text { pago aval BROU. }\end{array}$ \\
\hline $10-07-13$ & CG & CSS & PN-PC & MEF & Lorenzo & $\begin{array}{l}\text { Pluna S.A. Información acuerdo y } \\
\text { pago aval BROU. }\end{array}$ \\
\hline $12-06-13$ & LS & CSS & PN-PC & MSP & Muñiz & $\begin{array}{l}\text { Situación del Sistema Integrado } \\
\text { de Salud }\end{array}$ \\
\hline $05-03-13$ & LS & CSS & PN-PC & MEF & Lorenzo & $\begin{array}{l}\text { Medidas correctivas y resultados } \\
\text { económicos } 2013\end{array}$ \\
\hline 01-08-12 & LS & CSS & $\mathrm{PN}$ & MEC & Ehrlich & Acuerdo educativo \\
\hline 03-07-12 & LS & CSS & $\mathrm{PN}$ & MRREE & Almagro & Resoluciones del Mercosur. \\
\hline 09-05-12 & CG & CSS & PN-PC & MRREE & Almagro & Dragado del canal Martín García \\
\hline $02-05-12$ & LS & CRR & $\mathrm{PN}$ & MIN & Bonomi & Situación sistema carcelario \\
\hline $20-04-12$ & LS & CRR & $\mathrm{PN}$ & MIDES & Olesker & Programas sociales del MIDES \\
\hline $17-04-12$ & CG & CSS & PN-PC & MTOP & Pintado & Situación de Pluna \\
\hline $12-04-12$ & CG & CRR & Todos & MVOTMA & Muslera & Dirección de Medio Ambiente \\
\hline $11-04-12$ & CG & CSS & PN-PC & MEC & Ehrlich & Situación locales educativos \\
\hline $02-02-12$ & CG & $\mathrm{CP}$ & FA & MRREE & Almagro & Productos uruguayos en el exterior \\
\hline $02-02-12$ & CG & $\mathrm{CP}$ & FA & MIEM & Kreimerman & Productos uruguayos en el exterior \\
\hline $18-01-12$ & CG & $\mathrm{CP}$ & PN-PC & MIDES & Olesker & Adopción de menores INAU \\
\hline $11-01-12$ & CG & $\mathrm{CP}$ & PN-PC & MIN & Bonomi & Inseguridad pública \\
\hline
\end{tabular}

Notas: * Al 31 de julio de 2013; ver siglas de ministerios en Anexo I.

SIGLAS. LS: Llamados a Sala; CG: Llamados en Régimen de Comisión General; CRR: Cámara de Representantes; CSS: Cámara de Senadores; CP: Comisión Permanente;

Fuente: Chasquetti (2014). 
ción de haberse configurado en un asunto de campaña electoral, condimentado hacia fines de diciembre de 2013 con procesamientos sin prisión por abuso de funciones del ministro de Economía y el presidente del Banco República.

El segundo indicador fundamental en el control del ejecutivo por parte de la oposición son los pedidos de informe. Si bien el Cuadro 5 consigna datos para todo el periodo, obsérvese que los pedidos de informe muestran el predominio de ciertos tópicos. La mayoría de los pedidos de informe vinculados al Ministerio de Economía (MEF) están asociados al Pluna-gate. Sin embargo, y como vimos más arriba, el gobierno enfrenta algunos frentes problemáticos donde la oposición ha tratado de insistir públicamente, dando respuesta a grupos de interés y la opinión pública en general. En este sentido, la educación por el Ministerio de Educación (MEC), la minería de gran porte por el VOTMA, y el propio el Pluna-gate a través del Ministerio de Transportes (MTOP) figuran entre los ministerios que reciben el mayor número de pedidos de informes por parte de los partidos en el Parlamento. Por lejos, el Partido Nacional es el partido con el mayor número de informes, seguido por el Partido Colorado y la propia bancada de legisladores del partido de gobierno.

Cuadro 5. Pedidos de informes según partido: Uruguay 2010-2013*

\begin{tabular}{|c|c|c|c|c|c|c|c|c|}
\hline \multicolumn{3}{|c|}{ Frente Amplio } & \multicolumn{3}{|c|}{ Partido Colorado } & \multicolumn{3}{|c|}{ Partido Nacional } \\
\hline Destino & $\mathrm{N}$ & $\%$ & Destino & $\mathrm{N}$ & $\%$ & Destino & $\mathrm{N}$ & $\%$ \\
\hline MEF & 18 & 16 & MIEM & 82 & 10 & MIEM & 119 & 11 \\
\hline MIEM & 15 & 13 & MIN & 82 & 10 & MEC & 117 & 11 \\
\hline MEC & 11 & 10 & MSP & 81 & 10 & MVOTMA & 109 & 10 \\
\hline SCJ & 9 & 8 & MEC & 79 & 9 & MSP & 105 & 10 \\
\hline MTSS & 8 & 7 & MVOTMA & 77 & 9 & MEF & 87 & 8 \\
\hline MVOTMA & 8 & 7 & MEF & 74 & 9 & MIN & 72 & 7 \\
\hline MDN & 6 & 5 & MTOP & 56 & 7 & MTOP & 70 & 7 \\
\hline MTOP & 6 & 5 & MIDES & 48 & 6 & MIDES & 60 & 6 \\
\hline MGAP & 5 & 4 & $\mathrm{MDN}$ & 41 & 5 & MRREE & 56 & 5 \\
\hline ОРР & 5 & 4 & MRREE & 41 & 5 & MTSS & 55 & 5 \\
\hline MIN & 4 & 4 & MTSS & 33 & 4 & $\mathrm{MDN}$ & 43 & 4 \\
\hline ONSC & 4 & 4 & MGAP & 29 & 3 & MGAP & 39 & 4 \\
\hline ANEP & 3 & 3 & MTD & 29 & 3 & MTD & 28 & 3 \\
\hline MIDES & 3 & 3 & OPP & 13 & 2 & OPP & 14 & 1 \\
\hline MSP & 3 & 3 & ANEP & 7 & 1 & ONSC & 12 & 1 \\
\hline Otros & 4 & 4 & Otros & 64 & 8 & Otros & 80 & 8 \\
\hline Total & 112 & 100 & Total & 836 & 100 & Total & 1066 & 100 \\
\hline
\end{tabular}

Notas: * Al 31 de julio de 2013; ver siglas en Anexo I.

Fuente: Elaborado a base de datos del Sistema de Información Parlamentaria. 
En líneas generales, 2013 muestra una operativa del gobierno similar a la de años anteriores, con altas tasas de eficacia en la aprobación de legislación y una oposición particularmente proactiva en la solicitud de información y llamados de los ministros a sala, a los efectos de controlar la gestión del gobierno, hacerla pública y explotar políticamente algunos problemas del gobierno en áreas como la educación, la megaminería, la liquidación de Pluna y la seguridad pública.

\section{LA OPINIÓN PÚBLICA Y LA COMPETENCIA ELECTORAL}

Si se observa la serie de aprobación de la gestión del gobierno de José Mujica es posible advertir un importante deterioro en el primer año de gobierno y una caída moderada en el tercer año. En 2013 el presidente Mujica ha logrado mantener sus niveles de aprobación en forma relativamente estable. Si se comparan los niveles de aprobación de las gestiones de Mujica y Vázquez (ver Figura 2) no existen diferencias en la evolución de todo el periodo que comprenden los primeros cuatro años de gobierno. En otras palabras, el nivel de apoyo a las dos gestiones del Frente Amplio ha variado en forma casi idéntica. Sin embargo, lo que diferencia el estado de la opinión pública en los periodos es la intensidad con la cual los opositores desaprueban la gestión. Mujica comienza a tener mayores niveles de desaprobación que Vázquez desde el segundo año de gobierno. La Figura 2 muestra que a partir de ese entonces las diferencias de desaprobación comienzan a ser significativas.

Figura 2. Porcentajes de aprobación y desaprobación de la gestión del Presidente en los primeros cuatro años de gobierno. Gestiones de Tabaré Vázquez (20052009) y José Mujica (2010-2013)

$\%$ de aprobación

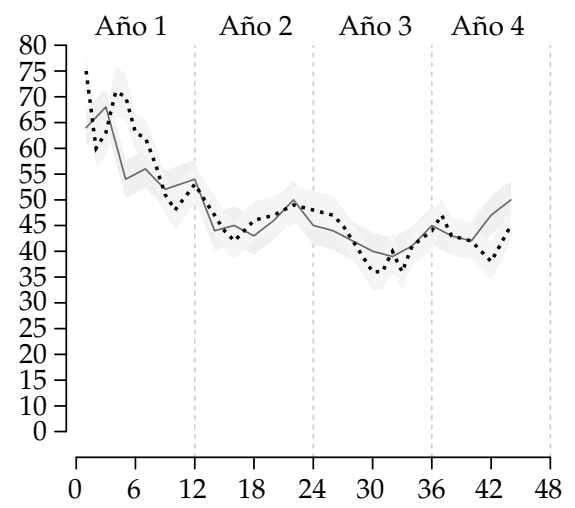

\% de desaprobación

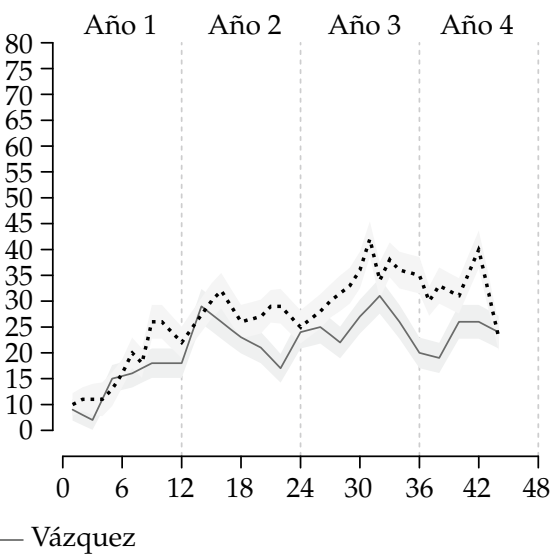

Nota: El área gris representa el intervalo de confianza del 95\% dadas las proporciones estimadas y los tamaños de las muestras en cada encuesta de opinión. Datos recolectados por el Banco de Datos del Instituto de Ciencia Política, Universidad de la República. 
No obstante, los ciclos de aprobación de la gestión del gobierno parecen no estar relacionados con las preferencias electorales. Más aún, cuando parece claro que existe un generalizado descontento en ciertas áreas de política pública como la educación, la seguridad ciudadana (criminalidad), el nivel de inflación en la vivienda y los alimentos, el partido de gobierno parece no sufrir consecuencias políticas visibles de cara a las próximas elecciones nacionales de 2014. Esto puede deberse a distintos factores. Primero, los uruguayos son personas con preferencias políticas relativamente estables, tanto en términos ideológicos como de adhesión a los partidos. Segundo, uno de los aspectos más relevantes para explicar el voto al oficialismo en cualquier democracia es el desempeño de la economía. A pesar de la desaceleración del crecimiento del producto, el desempeño de la economía ha sido positivo, el desempleo se mantiene estable y el salario real ha crecido durante todo el periodo. Tercero, el partido de gobierno (Frente Amplio) ofrece la candidatura del expresidente Tabaré Vázquez quien cuenta con altos niveles de popularidad y no se encuentra demasiado asociado a los problemas de la gestión del actual presidente José Mujica. Estos factores ayudan a desvincular consideraciones coyunturales de las preferencias electorales de mediano y largo plazo que caracterizan al electorado uruguayo.

La Figura 3 muestra los niveles de intención de voto desde octubre de 2009 hasta agosto de 2013. Ninguno de los cuatro partidos muestra cambios dramáticos. Los partidos tradicionales (Blanco y Colorado) recogen un apoyo similar a la intención de voto esperada para el Frente Amplio en todo el periodo. Esto sugiere incertidumbre respecto del resultado de las próximas elecciones nacionales en dos sentidos. En primer lugar, no hay certeza de si habrá o no una segunda vuelta electoral entre el candidato presidencial del Frente Amplio (Tabaré Vázquez, quien tiene una interna prácticamente asegurada) y el candidato del segundo partido más votado, seguramente el Partido Nacional. En segundo lugar, en la eventualidad de un tercer gobierno del Frente Amplio, tampoco no hay certidumbre sobre la composición del Parlamento, en especial sobre la existencia de las mayorías parlamentarias propias.

\section{EL CICLO ELECTORAL Y EL NACIMIENTO DE LA CONCERTACIÓN}

Uruguay reformó su constitución en 1996, produciendo varios cambios en la forma de elección del Presidente y el Legislativo, además de separar las elecciones municipales de las nacionales. De este modo se dio paso a la construcción de un largo ciclo que se inicia con las elecciones parlamentarias y presidenciales de octubre y alternativamente una posible segunda ronda presidencial en noviembre, seguido por la elección de autoridades municipales en mayo del siguiente año. Todo ello precedido por elecciones internas dentro de los partidos, cuyas implicaciones afectan todas las elecciones del ciclo. Curiosamente, la elección a nivel departamental (o local) es la última elección del ciclo y sin embargo es la que primero ha mostrado movimientos durante 2013.

La primera jugada del proceso electoral ha tenido lugar en el departamento de Montevideo, donde el Frente Amplio se ha constituido en partido dominante en la clásica categoría de Sartori (1976) luego de vencer en las pasadas cinco elecciones. Esta condición ma- 
Figura 3. Evolución de la intención de voto para las elecciones nacionales de 2014

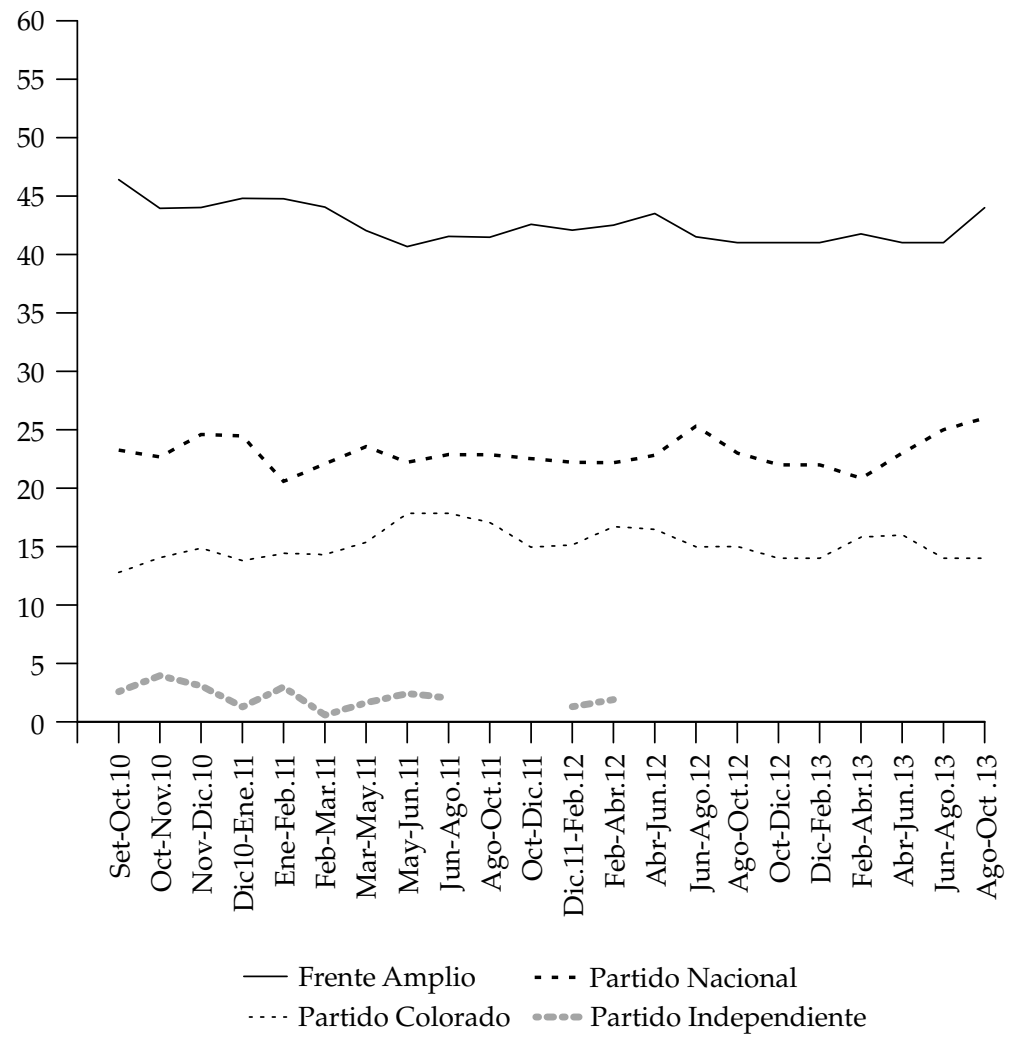

Fuente: Datos recolectados por el Banco de Datos del Instituto de Ciencia Política, Universidad de la República.

yoritaria aun la conserva en términos de intención de voto, ya que supera largamente a cualquiera de sus dos rivales tradicionales (blancos y colorados). Sin embargo, la última elección a nivel departamental dejó numerosas dudas a nivel del electorado frenteamplista de Montevideo, no solo a raíz de los resultados de la gestión, sino también a propósito del proceso de selección interna de su candidata (Ana Olivera). El Frente Amplio ganó, dejando un saldo de 13\% de votos en blanco, evidenciando una gran disconformidad entre votantes cautivos del Frente Amplio.

Con magros resultados de gestión y un gran desgaste al cabo de veinticinco años al frente del gobierno departamental, las chances frentistas de ganar nuevamente permanecían intactas, siempre y cuando blancos y colorados fueran incapaces de coordinar electoralmente una oferta unificada que les permitiera ganar (Cox, 1997). Precisamente la coordinación electoral implica (por definición) una reducción drástica en el número de candidatos, que, en el caso de una elección por mayoría simple-como es el caso a nivel departamental-, ronda en el entorno de los dos candidatos. Por encima de este nivel de equilibrio, tres candidatos muestran ineficiencia electoral para dos de los competi- 
dores en pugna. Por este motivo, blancos y colorados finalmente llegaron a un acuerdo en 2013 que les permite correr bajo un nuevo paraguas partidario, sin dejar de proponer sus propios candidatos (bajo el mismo paraguas). Este nuevo partido asentado en Montevideo se llama La Concertación y entraña un serio riesgo para la continuidad del Frente Amplio en el gobierno de Montevideo (Cardarello y Lorenzoni, 2013).

Como todo proceso de coordinación electoral, el acuerdo implicó un proceso de negociación complejo entre blancos y colorados. Nótese que este proceso de negociación no fue llevado a cabo por autoridades locales. Muy por el contrario, el Partido Nacional y el Partido Colorado designaron a dos senadores de gran reputación dentro de cada uno de sus partidos para conducir la negociación en búsqueda de un acuerdo que los llevara a la construcción de un partido común a nivel departamental (solo en Montevideo). El beneficio de la unión es evidente. El costo, sin embargo, es echar por tierra las ambiciones de algunos potenciales candidatos dentro de cada uno de los partidos, debido a que el tamaño máximo de la oferta no puede superar a los tres candidatos dentro de la Concertación. Asimismo, este número fuerza a los dos partidos del acuerdo a no tener mayoría de candidatos. Esto es, el tercer candidato debe ser independiente: un candidato reputado pero sin adscripción partidaria claramente identificable. Este dilema está en curso y no es de fácil dilucidación.

El segundo escollo que buscan evitar las élites partidarias es que el acuerdo, independientemente de su éxito electoral en mayo de 2015, se difunda a lo largo y a lo ancho del territorio. La configuración de Montevideo, donde un partido domina la escena y alguno de los partidos de oposición se ve minimizado progresivamente, es una constante en numerosos departamentos del país. El ejemplo de coordinación electoral exitoso en Montevideo es visto con muy buenos ojos por representantes locales que ven sus chances de acceder al gobierno como un hecho remoto o improbable. Inevitablemente, los resultados de la elección departamental de Montevideo en 2015 servirán de ejemplo para las élites o los dirigentes locales, a los efectos de extender la coordinación electoral o mantener el statu quo.

\section{CONSIDERACIONES FINALES}

En este artículo hemos revisado los principales acontecimientos políticos y económicos de lo sucedido en Uruguay durante el 2013. En materia económica el producto bruto ha continuado creciendo a un ritmo similar al de 2012, el poder adquisitivo de los hogares ha aumentado, y las presiones inflacionarias representan el mayor signo de preocupación macroeconómica.

En materia de políticas públicas la principal novedad ha sido la agenda de derechos impulsada por el gobierno. El matrimonio igualitario, el derecho a la interrupción del embarazo, y la regulación de la Cannabis son innovaciones de política pública que el gobierno ha impulsado al margen del programa del Frente Amplio. El 2013 también dejó resultados negativos. La evolución de los niveles de cobertura del sistema educativo en secundaria no muestran mejoría, y los resultados de aprendizaje empeoran 
(a pesar de los niveles de gasto en este sector). Por otra parte, ha crecido el descontento con la seguridad pública y se ha cristalizado el fracaso de la política de vivienda impulsada por el Presidente.

En materia política, los escándalos en el seno del gobierno a causa del caso Pluna le han costado al gobierno la pérdida del ministro de economía, el ministro que hasta ese entonces gozaba de la mayor reputación dentro del gabinete. A pesar de ello, la aprobación de la gestión del presidente Mujica se mantuvo estable a lo largo del año y los niveles de intención del voto para el partido de gobierno sugieren un escenario muy similar al que se dio en las últimas dos elecciones, donde la izquierda obtuvo el poder y se aseguró mayorías parlamentarias. No obstante, el panorama electoral hacia 2014 está completamente abierto.

\section{REFERENCIAS}

Amorim Neto, Octavio. 1998. Cabinet Formation in Presidential Regimes: an Analysis of 10 Latin American Countries. Presentado en la conferencia anual de Latin American Association, Illinois.

Buquet, Daniel y Diego Luján. 2013. "Actividad del Poder Ejecutivo". En Informe de Coyuntura del Departamento de Ciencia Política, ed. G. Caetano, Juan A. Moraes y José M. Busquets, 95-110.

Cardarello, Antonio y Miguel Lorenzoni. 2013. "El Escenario Subnacional a Mitad de Camino". En Informe de Coyuntura del Departamento de Ciencia Política, ed. G. Caetano, Juan A. Moraes y José M. Busquets.

Chasquetti, Daniel. 2014. Informe final al Poder Legislativo. Technical report Universidad de la República.

Chasquetti, Daniel y Alejandro Guedes. 2013. "La Actividad Legislativa en los Albores de la Carrera Electoral". En Informe de Coyuntura del Departamento de Ciencia Política, ed. G. Caetano, Juan A. Moraes y José M. Busquets, 111-132.

CINVE. 2013. Pilares del Crecimiento. Actividad y Comercio No 119. Centro de Investigaciones Económicas.

Cox, Gary W. 1997. Making Votes Count: Strategic Coordination in the World's Electoral Systems. Cambridge University Press.

IECON. 2013a. El Tipo de Cambio Real Como Indicador de la Competitividad. Observatorio de coyuntura. boletín $\mathrm{N}^{\circ} 1$. Instituto de Economía. Universidad de la República.

IECON. 2013b. Inflación en Uruguay: evolución y medidas. Observatorio de coyuntura. boletín $\mathrm{N}^{\mathrm{o}} 2$. Instituto de Economía. Universidad de la República.

Monestier, Felipe. 2013. "El Partido de Gobierno". En Informe de Coyuntura del Departamento de Ciencia Política, ed. G. Caetano, Juan A. Moraes y José M. Busquets, 149-158.

Sartori, Giovanni. 1976. Parties and Party Systems. A Framework for Analysis. New York: Cambridge University Press.

Juan Andrés Moraes es Ph.D. en Ciencia Política (Notre Dame, 2010) y es profesor adjunto en el Departamento de Ciencia Política en la Universidad de la República. Actualmente forma parte del área de Gobierno y Partidos Políticos y sus publicaciones han aparecido en libros y revistas nacionales e internacionales. Es coautor con Daniel Buquet y Daniel Chasquetti del "Enfermo Imaginario" (FCS, 1998), coeditor con Diego Aboal de "Economía Política en Uruguay" (Trilce, 2003), mientras que otros trabajos más recientes han sido publicados en Penn State University Press (2008), Harvard University Press (2009), Comparative Political Studies (2009) y Party Politics (2012). E-mail: Juan.A.Moraes.1@nd.edu

Santiago López Cariboni es Ph.D. en Ciencia Política por la Universidad de Essex, Reino Unido. Actualmente es profesor asistente en el Departamento de Ciencias Sociales y Políticas, Universidad Católica del Uruguay. Es además Director de la Escuela de Invierno en Métodos y Análisis de Datos UCU-DCSP. E-mail: santiago.lopez@ucu.edu.uy 
